\title{
Topical Airway Anaesthesia Techniques In Dental Patients With Temporomandibular Joint Ankylosis: A Comparison Between Nebulization Versus Nerve Blocks For Awake Nasal Fiberoptic Intubation
}

\author{
Dr PriyankaBansal ${ }^{1}$, DrML Khatri ${ }^{2}$ \\ Department of anaesthesiology and Intensive care, SGT University, Gurgaon, Haryana \\ Assistant Professor ${ }^{1}$, Associate Professor ${ }^{2}$
}

\begin{abstract}
Introduction:Awakenasal fiberoptic bronchoscope (FOB) guided intubation is the gold standard of airway management in patients withtemporomandibular joint ankylosis. It is essential to sufficiently anesthetize the upper airway before the performance of awake FOB guided intubation in order to ensure patient comfort and cooperation and maintain haemodynamics. This randomized controlled study was performed to compare two methods of airway anesthesia, namely topical nebulization of local anesthetic and performance of airway blocks.

Materials and methods: Consent was obtained from 46 adult patients of temporomandibular joint ankylosis, scheduled for gap arthroplasty. They were enrolled for thisdouble-blind, randomized, prospective clinical trial with two treatment groups - Group N and Group NB, of 23 patients each. Group N received airway anesthesia through jet nebulization of $10 \mathrm{ml}$ of $4 \%$ lignocaine and Group NB received airway blocks (bilateral superior laryngeal and transtracheal recurrent laryngeal) each with $2 \mathrm{ml}$ of $2 \%$ lignocaine aswell as topicalairway spray with $4 \%$ lignocaine. FOB guided nasotracheal intubation was then performed. Hemodynamic variables at baseline and during the procedure, patient recall and comfort- before and after intubation, vocal cord visibility, conditions achieved at endoscopy, coughing/gagging episodes, and postoperative events were noted.

Results:The success rate of awakefiberoptic intubation was 100\%.The haemodynamic parameters, endoscopic conditions and patient response and comfortwas better with nerve block group than nebulization group. Most of the intubations were carried out in less than 3 minutes, however the time taken in group NB was less than in group $N$. Vocal cord visibility and ease of intubation were better in patients who received airway blocks and hence the amount of supplemental lignocaine used was less in this group.Conclusion: Both ways of topical anaesthesia of airway nebulisation with lignocaine and nerve block technique are satisfactory. However fiberoptic nasal intubation using nerve block technique provides better intubating conditions, patient satisfaction, haemodynamic stability with minimal complications.
\end{abstract}

\section{Introduction}

Ankylosis of temporo mandibular joint is a clinical condition in which there is bony and/or fibrous fusion of mandibular condyle with glenoid fossa of temporal bone. Factors for this include trauma, local and systemic infections as well as systemic disease like rheumatoid arthritis, psoriasis and ankylosing spondylitis. ${ }^{1,2,3}$ In these patients there is no mouth opening or mouth opening is grossly restricted which challenge anaesthetist for difficult airway management. Nasotracheal intubation is necessary in patients suffering from temporo-mandibular joint ankylosis. Various techniques are used for nasotracheal intubation. ${ }^{4}$

In recent years, there have been many advances in airway management.One major decision must be made with every procedure: will the patient beintubated while under general anaesthesia, or does the patient need to beawake during intubation? The American Society of Anesthesiologists (ASA) and many European authors recommend awake fiberoptic intubation where difficult intubation is anticipated, which can lead to the life-threatening "can't intubate, can't ventilate scenario." Although awake intubation is much more time consuming for the anaesthesiologist and a more unpleasant experience for the patient,there are several compelling reasons why intubation should be done while patient with a recognised difficult airway is still awake. First, and most important, the natural airway will be better maintained in most patients when they are awake ("no bridges are burned"). Second in the awake patient enough muscle tone is maintained to keep the relevant upper airway structures (the base of tongue, valleculla, epiglottis, oesophagus and posterior pharyngeal wall) separated from one another and much easier to identify. In the anaesthetized and paralized patient, loss of muscle tone tends to cause these structures to collapse towards one another (e.g. the tongue moves posteriorly), which distorts the anatomy. Third the larynx moves to a more anterior position with the induction of anaesthesia and paralysis, which makes conventional intubation more difficult. Thus if a difficult intubation is anticipated, 
awake tracheal intubation is often indicated. Crucial to the success of an awake tracheal intubation is proper preparation of the patient; most techniques will work well in most patients when they are quite and cooperative and have a larynx that is nonreactive to physical stimuli. ${ }^{6,7}$

Direct laryngoscopy in an awake, unprepared patient can be extremelychallenging. Excessive salivation and gag and cough reflexes can makeintubation difficult, if not impossible, under awake conditions. In addition,the stress and discomfort may lead to undesirable elevations in the patient'ssympathetic and parasympathetic outflow. ${ }^{8}$

Several highly effective topicaland regional anaesthesia techniques have been developed to subdue thesereflexes and facilitate intubation. This can be achieved by either (a) Topical administration of local anesthetic (LA) or (b) blockade of neural supply to oropharynx and larynx. Gupta et al. in 2014 conducted study on 50 patients with cervical spine injury allocated in two groups one receiving airway anesthesia through ultrasonic nebulization of $10 \mathrm{ml}$ of $4 \%$ lignocaine and other receiving airway nerve blocks (bilateral superior laryngeal, transtracheal, and recurrent laryngeal nerve). ${ }^{10}$ Nerve block group showed less time taken for intubation, less number of coughing/gagging episodes, more vocal cord visibility and more ease of intubation and less use of extra LA as compared to the nebulized group. This study formed the basis of our research. We comparedawakefiberoptic intubation in 46 adult patients with TM joint ankylosis having Mallampatti grade III and IV posted for surgery scheduled to undergo nasal intubation. Patients were divided into two groups- Gp N received lignocaine nebulisation through facemask using a micronebulizer with oxygen @10 L/min, Gp NB. received airway blocks (bilateral glossopharyngeal,bilateral superior laryngeal and transtracheal recurrent laryngeal) each with $2 \mathrm{ml}$ of $2 \%$ lignocaine aswell as topical airway spray with $4 \%$ lignocaine.

\section{Materials And Method}

After approval by the institutional ethics committee, the protocol was conducted in 46 patients of either sex, age 18-60 years, ASA physical status grade I and II and Mallampatti grade III and IV a written informed consent was taken from each patient. All patients who were assessed at preoperative evaluation to be potentially difficult to intubate, difficult to ventilate, MP grade III and IV, thyromental distance of less than $5 \mathrm{~cm}$ and interincisor distance of less than $1.5 \mathrm{~cm}$ or have past history of difficult or failed tracheal intubations were selected for awake fiberopticnasotracheal intubation (AFNI). Exclusion criteria were nasal problems(fractures, trauma), fracture base of the skull, lower respiratory tract hypersensitivity (asthma, bronchitis), allergy to drugs used in the procedure (lignocaine), epileptics, bleeding tendency, obesity and endocrine problems and on drugs known to interfere with heart rate and blood pressure such as $\beta$ blockers, calcium channel blockers.

Standard fasting guidelines and anti-aspiration prophylaxis with tablet ranitidine $150 \mathrm{mg}$ were prescribed. The patients were explained about the awake FOB guided intubation during preoperative assessment. Nasal xylometazoline drops ( 2 in each nostril, 3 times at a gap of half hour) were given by the residents in the morning of procedure. Injection glycopyrrolate $5 \mu \mathrm{g} / \mathrm{kg}$ was given intramuscularly half an hour before shifting the patient to the operating room (OR). Inside the OR, standard monitoring, including electrocardiography (ECG), noninvasive blood pressure (BP), and pulse oximetry $\left(\mathrm{SpO}_{2}\right)$ were applied in all patients. An intravenous (IV) line was secured and ringer lactate was started.Two lignocaine(2\%) soaked cotton plegets were placed in both the nostrils (upto $1 \mathrm{ml}$ in each nostril. After recording the baseline heart rate (HR), $\mathrm{BP}$ and $\mathrm{SpO}_{2}$, injection midazolam (1-2 mg)and injection nalbuphine $0.3 \mathrm{mg} / \mathrm{kg}$ were given IV. Doses of both drugs, however, were further restricted by judgement of the anaesthesiologist with the goal that all patients would remain awake and able to follow commands throughout the procedure.

The patients were randomly allocated into two groups. Randomization was done using computer generated tables of random numbers.

1. Topical anaesthesia group $(\mathrm{Gp} \mathrm{N}, \mathrm{n}=23)$ - A nebulizer(Orane, made in Germany)with the oxygen flow rate of $10 \mathrm{~L} / \mathrm{min}$ was used to deliver upto $10 \mathrm{ml}$ of $4 \%$ lignocaine (lignocaine hydrochloride, Neon laboratories) into the oropharynx. Patients were encouraged to inhale deeply through their mouth while their nose was plugged (with lignocaine soaked plegets) to facilitate entrainment of nebulized lidocaine into their airway over $10 \mathrm{~min}$.

2. Nerve block group $(\mathrm{Gp} \mathrm{NB}, \mathrm{n}=23$ ) lignocaine spray(4\%) was applied directly to the tongue (50 mg total). The lingual branch of glossopharyngeal nerve was blocked bilaterally by infiltrating the inferior aspect of the palatoglossal arch with $0.5-1 \mathrm{ml}$ of $2 \%$ lignocaine. The superior laryngeal nerves were blocked bilaterally by infiltrating $2 \%$ lidocaine (1-2 ml) at the lateral and inferior aspect of hyoid. Finally $4 \%$ lidocaine $(3 \mathrm{ml})$ was injected through the cricothyroid membrane into the trachea.

Adequate effect of local anesthesia was confirmed by heaviness of tongue in Group $\mathrm{N}$ patients and by hoarseness of voice in Group NB patients. To maintain uniformity, airway anaesthesia or sedation for all patients was provided by one of the two anaesthesiologists (one of the consultants). After the transtracheal injections, patients in both the groups were treated identically. 
While giving supplemental oxygen through nasal prongs, FOB guided intubation was performed. Size $7.5 \mathrm{~mm}$ internal diameter endotracheal tube was used for male patients and $7 \mathrm{~mm}$ for female patients. Vital parameters ( $\mathrm{HR}, \mathrm{BP}$, and $\mathrm{SpO}_{2}$ ) were also recorded during intubation and at 1 min and 3 min postintubation. Supplemental LA was given as $1 \mathrm{ml}$ aliquots of $2 \%$ lignocaine through the working channel of FOB, "spray as you go" technique (next aliquot given only after waiting for 30-60 s). The total dose of lidocaineadministerd was recorded. Bronchoscopy time (from insertion of the fiberoptic bronchoscopy in the nostril to visualization of the carina), intubation time (insertion of tracheal tube into the nose to confirmation of tracheal intubation with capnograph), any hypoxic episode $\left(\mathrm{SPO}_{2}<90 \%\right)$, number of attempts at intubation and were noted.

Other parameters such as gag/cough reflex, cord visibility (relaxed, partially relaxed or adducted on endoscopic view), and ease of intubation [Table 1] were also recorded. Postoperative adverse events of hoarseness and sore throat, any signs of lignocaine toxicity such as ECG changes, seizures, and bronchoconstriction were also noted. After the airway was secured, general anesthesia was administered with propofol $2 \mathrm{mg} / \mathrm{kg}$, and vecuron 0.1 $\mathrm{mg} / \mathrm{kg}$.

The quality of anaesthetic during intubation was graded by an observer who was blinded to the anaesthetic group assignment. The intubation grading scale was defined as follows.

Table 1: Intubation Grading Scale

\begin{tabular}{|l|l|}
\hline 0 & No coughing or gagging in response to intubation \\
\hline 1 & Mild coughing and/or gagging that did not hinder intubation \\
\hline 2 & Moderate coughing and/or gagging that interfered minimally with intubation \\
\hline 3 & Severe coughing and/or gagging that made intubation difficult \\
\hline 4 & $\begin{array}{l}\text { Severe coughing and gagging that required additional local anaesthesia and/or other change in } \\
\text { technique to achieve successful intubation. }\end{array}$ \\
\hline
\end{tabular}

Subsequent anaesthetic techniques were managed as required for the surgical procedure. Vital parameters $\left(\mathrm{HR}, \mathrm{BP}, \mathrm{SpO}_{2}\right)$ were recorded at an interval of 1 min until intubation was done followed by every 2 min until $10 \mathrm{~min}$ after intubation. Other parameters recorded were patient's comfort score [Table 2] and, intubation time, intraoperative complications, and finally patient's satisfaction score postoperatively.

TABLE 2 Patients comfort score intraoperatively and satisfaction score postoperatively

\begin{tabular}{|l|l|l|l|}
\hline & $\begin{array}{l}5 \text { point fibroptic intubation comfort } \\
\text { score }\end{array}$ & $\begin{array}{l}\text { 3 point score immediately after } \\
\text { nasotracheal intubation }\end{array}$ & $\begin{array}{l}\text { Patient satisfaction score } \\
\text { postoperatively }\end{array}$ \\
\hline 0 & No reaction & Cooperative & Complete amnesia \\
\hline 1 & Slight grimacing & Restless/minimal resistance & Partial recall \\
\hline 2 & Heavy grimacing & Severe resistance & Unpleasant \\
\hline 3 & Verbal objection & & \\
\hline 4 & Defensive movements of head or hands & & \\
\hline
\end{tabular}

\section{Statistical Analysis}

All data were tabulated and analyzed statistically using software SPSS 17.0. Parametric values were reported as mean \pm standard deviation. Hemodynamic variables were compared using the unpaired Student's $t$ test. Intubation grades and patient comfort scores were compared using the Mann - Whitney $U$ test. Statistical significant value was considered if $P<0.05$.the power of the study was calculated using patient comfort score and intubation grading score and came out to be $>80 \%$.

\section{Results}

The demographic data [Table 2] showed no significant differences between the two groups. There was no statistically significant difference between both groups at any interval for HR or BP [Figure 1]. Patients in both groups exhibited a slight decrease in $\mathrm{SpO}_{2}$ during the procedure, but the lowest $\mathrm{SpO}_{2}$ recorded was $94 \%$. All the patients remained sufficiently awake to cooperate with the procedure and none of the patients showed any evidence of lignocaine toxicity.

Awake nasal FOB guided intubation was accomplished in all patients in both groups and in no patient was the procedure abandoned due to discomfort. The time taken to perform FOB guided intubation was less in Group NB (130.41 $\pm 55.67 \mathrm{~s})$ as compared with Group N (210.03 $\pm 47.88 \mathrm{~s})$ and this was statistically significant [Table 2]. Significantly more number of patients experienced gag and coughing during the procedure in Group $\mathrm{N}$ as compared with Group NB as seen by intubation grading scale[Figure 2]. It was due to this reason that supplemental lignocaine had to be used in significantly more number of patients in Group $\mathrm{N}$ as compared with Group NB $(n=14$ in Group N as compared to $n=5$ in Group NB, $P=0.009)$. The mean supplemental lignocaine volume used was $2.53 \pm 0.85 \mathrm{ml}$ and $0.86 \pm 0.34 \mathrm{ml}$ in Group N and Group NB, respectively. This difference was statistically significant $(P<0.001)$. The highest quantity of supplemental lignocaine used in 3 patients was $5 \mathrm{ml}$ in Group N $(n=3)$ and $4 \mathrm{ml}$ in Group NB $(n=2)$. 
Topical Airway Anaesthesia Techniques In Dental Patients With TemporomandibularJoint ..

The vocal cord visibility [Figure 3] was better in Group NB as compared to Group N $(P<0.001)$. Only 8 patients in Group $\mathrm{N}$ had completely relaxed vocal cords as opposed to 15 patients in Group NB. Partially relaxed vocal cords (moving,closing) were observed in 15 patients in Group $\mathrm{N}$ and 8 patients in Group NB. Completely adducted vocal cords were seen in only one patient in Group N. in this patient more amount of supplemental lignocaine was given. There was no statistical difference in between the two groups regarding the intubating conditions $(P>0.05)$ [figure 3]. Patient comfort was also much better in nerve block group and hence patient cooperation was more in this group [figure 5,6]. Only seven patients in Group N reported complete amnesia as compared to 10 patients in Group NB. No patient in Group NB reported recall of unpleasant memories as compared with five patients in Group $\mathrm{N}(P=0.008)$. Other complications such as pain in the throat, haematoma formation at the site of injection etc. were insignificant in both the groups.
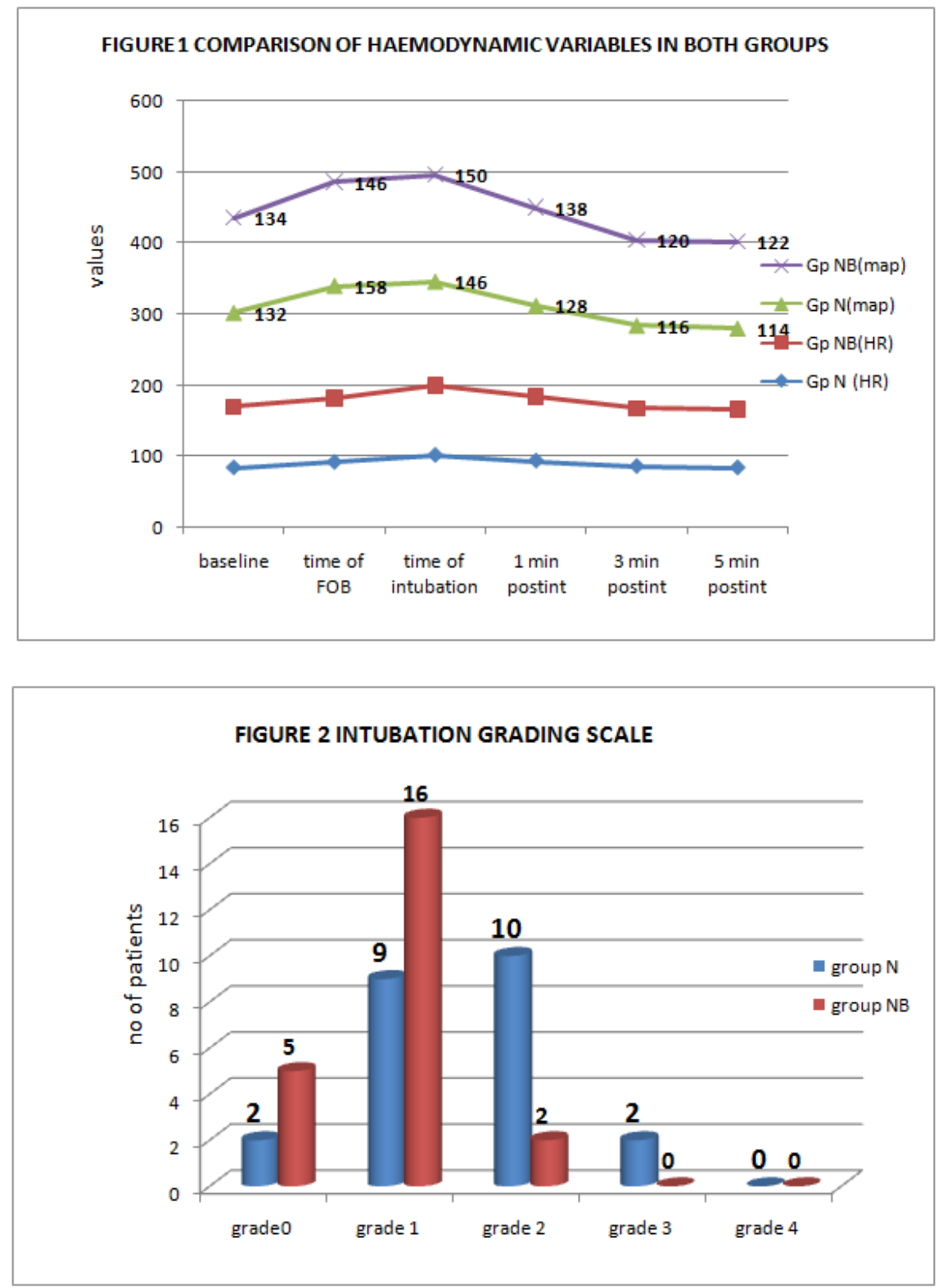

Table 2: Demographic Data And Time Taken For Intubation

\begin{tabular}{|l|l|l|l|}
\hline & Gp N & Gp NB & P value \\
\hline Age (in years) & $42.1 \pm 1.45$ & $39.27 \pm 4.96$ & .83 \\
\hline Sex (male/female) & $19 / 4$ & $21 / 2$ & \\
\hline Weight (in kg) & $58.23 \pm 8.83$ & $60.17 \pm 7.43$ & .67 \\
\hline Time for FOB(s) & $210.03 \pm 47.88$ & $130.41 \pm 55.67$ & 0.024 \\
\hline ASA grade (I/II) & $14 / 9$ & $16 / 7$ & \\
\hline FOB : fiberoptic bronchoscopy & \\
\hline
\end{tabular}



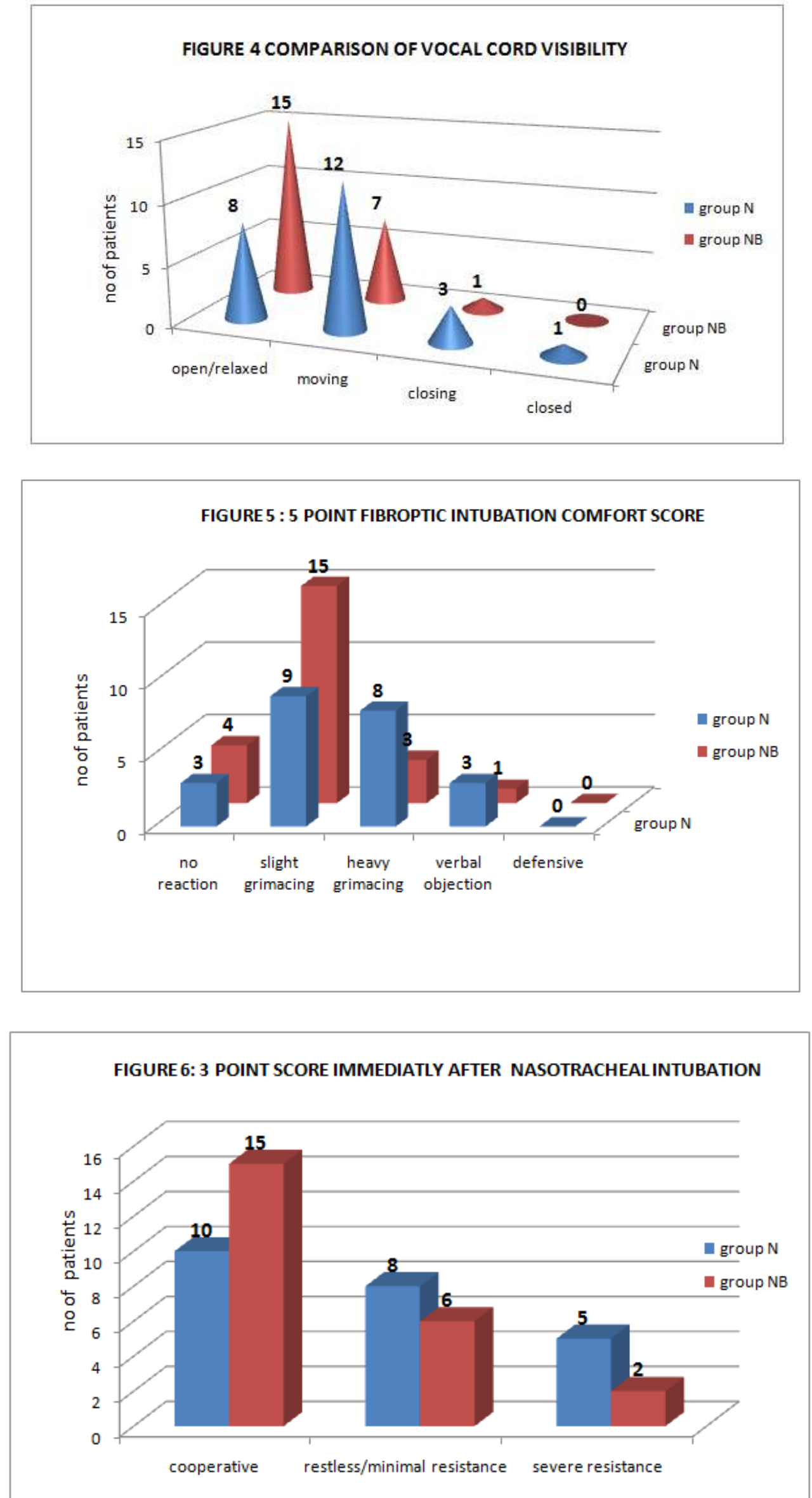


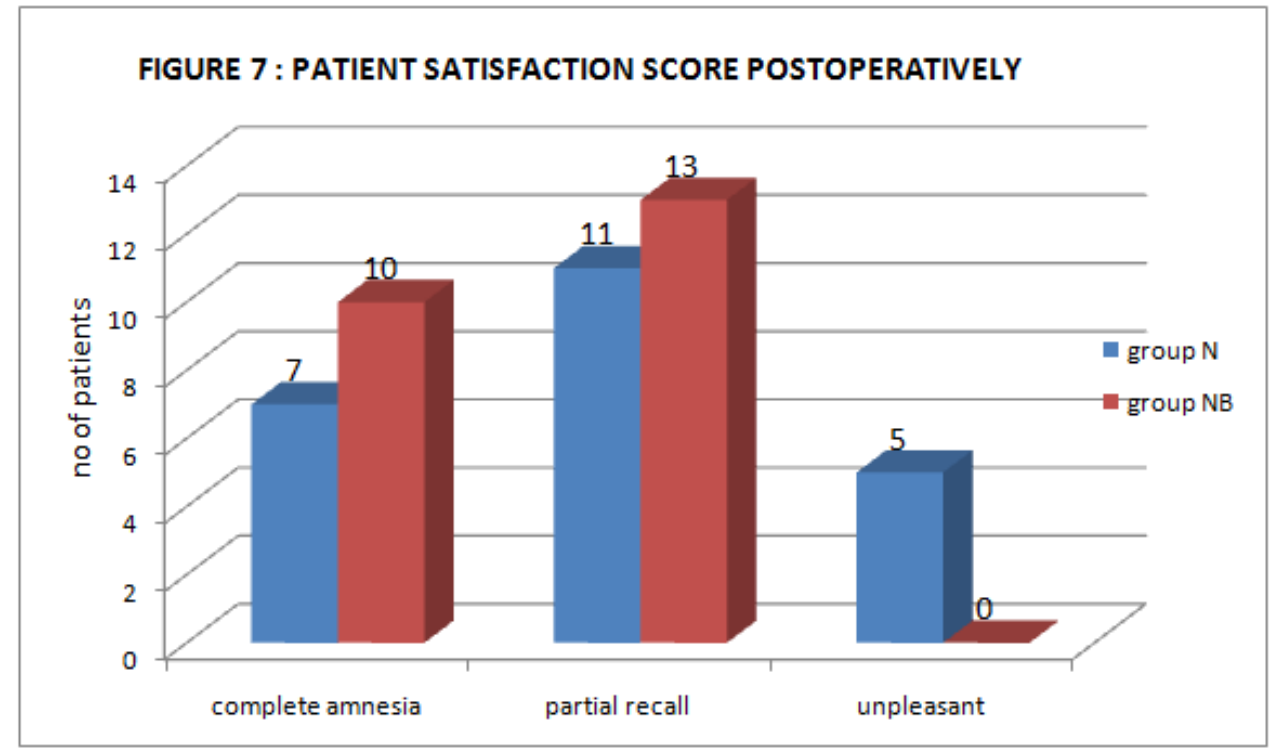

Table 3: Comparison Of Complications In Both Groups

\begin{tabular}{|l|l|l|l|}
\hline & COMPLICATIONS & Gp N & Gp NB \\
\hline 1 & Traumatic nasal bleed & 2 & 2 \\
\hline 2 & Bronchospasm & nil & nil \\
\hline 3 & Laryngospasm & nil & nil \\
\hline 4 & Painful nose & 1 & nil \\
\hline 5 & Postoperative sore throat & 1 & 2 \\
\hline 6 & Desaturation during procedure & nil & 1 \\
\hline
\end{tabular}

\section{Discussion}

Airway management is core for anesthesiologist. Deficiency in training or equipment as well as complications in this field are responsible for a significant proportions of anesthesia associated morbidity and mortality. Nevertheless the cannot ventilate cannot intubate scenario still occurs and regularly results in poor outcome such as permanent neurological deficit or even deaths. Therefore fibre optic intubation remains gold standard in patients with limited mouth opening and difficult airway because when applied correctly this technique never leads to point where patients' respiration is compromised. ${ }^{11}$

Haemodynamic responses, airway injuries, hypoxia, hypercarbia, bronchospasm and respiratory depression are well recognized hazards of patients during awake nasal intubation. ${ }^{12}$ Awake tracheal intubation with the aid of a fiberoptic device was first described by Murphy in 1967, who used a choledochoscope to facilitate nasotracheal intubation in patients with difficult airway. ${ }^{13}$ Since then, numerous subsequent authors have described the anesthetic techniques and experiences with awake FOB guided intubation.

There are multiple ways of anesthetizing the airway to facilitate the performance of awake FOB guided intubation. Among them, topical anesthesia with nebulized LA, gargles, lozenges, sprays, airway blocks and LA through the working channel of FOB is commonly used. Although the above-mentioned techniques can be combined in various ways, we chose two mutually exclusive techniques to compare their efficacy and patient comfort.

In the present study, the authors topicallyanesthetized the anterior ethmoidal nerve and sphenopalatineganglion by packing the selected nostril with $1 \mathrm{ml}$ of $2 \%$ lignocaine on cotton pledgets into the nasalcavity and anesthetized the pharynx, larynx and trachea with $10 \mathrm{ml}$ of $4 \%$ lidocaine nebulized with a micronebulizer gas through a standard face mask for 10 minutes with supplement of $2 \%$ lidocaine $2-3 \mathrm{ml}$ via bronchoscope. ${ }^{14} \mathrm{~A}$ recent study by William et al which used nebulisation technique and supplement with the spray-as-you-go technique also had similar successful results. ${ }^{15}$

Intubation with fiberoptic bronchoscope is easier in a conscious patient than the anesthetized one, whose decreased tone of the tongue, epiglottis, pyriform fossa and muscles and result in a sagging posterior tongue, falling back epiglottis and relaxing the pyriform fossa ${ }^{16}$.

Awakefiberoptic intubation via nasal approach is often easier than the oral approach because the fiberoptic bronchoscope is usually pointed straight at the glottis as it entered the oropharynx, there is no sharp turn to negotiate and the vocal cords are usually visible from a distance. The causes of failure in AFNI are poor topical anesthesia that made patients incorporative and with major nasal bleeding. ${ }^{17}$ 
In the present study, the authors chose a smalldiameter endotracheal tube (Portex soft seal; number 7.5 for male and number 7 for female) because most of the patients being included in the present study had narrow or distorted airways and in order to avoid the problem of trauma, such as nasal bleeding.

The mean time taken for endotracheal intubation was $2.12 \pm 0.12 \mathrm{~min}$. A study conducted by Gupta et al. also showed average time of $123.0 \pm 46.7 \mathrm{~s}$ taken for awake intubation under regional nerve blocks. ${ }^{10}$ In our study, the efficiency and adequacy of the blocks given were assessed by using Intubation scores which included (a) Vocal cord movements (b) Cough score (c) gag reflex which showed successful application of block in around $90 \%$ of patients. The further comfort of the patient was assessed by using 5-point patient comfort score during the procedure and 3-point comfort score after awakenasotracheal intubation. In a study done by Chatrath et al on the efficacy of combined regional nerve blocks the mean time taken for endotracheal intubation was $2.12 \pm 0.12 \mathrm{~min}$. A similar intubation grading scale and patient comfort score was used and it was concluded that awake fiberopticorotracheal intubation done under adequate LA given by combined regional nerve blocks is associated with good intubating conditions and patient comfort with minimal effect on in hemodynamics. ${ }^{28}$

In 2001, the British Thoracic Society recommended the total dose of lidocaine applied duringbronchoscope should be limited to $8.2 \mathrm{mg} / \mathrm{kg}$. ${ }^{18}$ Dosesof up to $14.77 \mathrm{mg} / \mathrm{kg}$ of lidocaine, which were administeredby a spray-as-you-go method, were reported tohave experienced involuntary movement symptoms thatindicated cortical irritability leading to a convulsion. ${ }^{19}$ Although, in 1990 , Webb J. measured serial plasmalidocaine concentrations during a similar protocol fortopical lidocaine nebulizer and reported very low systemicabsorption of topical lidocaine and minimal cardiovasculareffect. ${ }^{20}$ Similarly, Langmacket al. measured the serum lignocaine levels in 51 asthmatic volunteers undergoing FOB with topical lignocaine. ${ }^{22}$ The average total dose used was $600 \mathrm{mg}(8.2 \mathrm{mg} / \mathrm{kg})$, which was found to be safe in all patients as assessed by serum lignocaine concentrations. However, in 1993, Wu et al. have reported seizures in a patient after administration of a total dose of $300 \mathrm{mg}$ of topical lignocaine during FOB. ${ }^{22}$ The serum lignocaine concentrations were found to be well above the acceptable toxic limits. Hence, a constant lookout for signs and symptoms of lignocaine toxicity is mandatory while using large doses.

Inthe present study, the total dose of topical lidocainewas $7-8 \mathrm{mg} / \mathrm{kg}$ (median dose $7.54 \mathrm{mg} / \mathrm{kg}$ in nebulisation group and $5.88 \mathrm{mg} / \mathrm{kg}$ in nerve block group) in both the groups whichis in accordance with previous studies and guidelinesand the authors found no symptom of local anesthetictoxicity.

Through this technique, we blocked the three major reflexes of the patients including gag reflex, cough reflex, and glottis closure reflex by blocking bilateral glossopharyngeal, bilateral superior laryngeal, and recurrent laryngeal nerve, respectively. In our study, we observed an increase in HR, SBP, DBP and mean arterial pressure during the procedure of fiberoptic intubation (maximum seen at the time of tracheal intubation) which later on settled until the $3^{\text {rd }}$ to $4^{\text {th }}$ min after intubation was done, which was similar to that observed by Ovassapianet al. while performing nasotracheal intubation in awake patients under LA. ${ }^{23}$ In a study conducted by Trivedi and Patil in 2009 in which they evaluated airway blocks versus general anesthesia concluded that hemodynamic changes were less in airway block patients. ${ }^{9}$

Administration of lignocaine through nebulization for anesthesia of upper airway and larynx has also been previously studied.In 2007, Techanivateet al.found adequate upper airway anesthesia with $2 \%$ lignocaine nebulization and topical cocaine application to the nose for fiberopticnasotracheal application. ${ }^{24}$

In our study, the time taken to perform FOB guided intubation was significantly more in the nebulization group as compared to the nerve blocks group. This is in contrast to the randomized double-blinded study conducted in 1995 by Reasoneret al., which compared nebulized lignocaine with airway blocks to aid in FOB guided intubation in patients with cervical spine instability. ${ }^{25}$ The topical anesthesia group received $20 \mathrm{ml}$ of $4 \%$ lignocaine via nebulization followed by a $3 \mathrm{ml}$ transtracheal injection. On the other hand, the nerve block group received bilateral glossopharyngeal and superior laryngeal nerve blocks along with the transtracheal injection. They found no significant difference in the time taken to intubate between the groups. This was probably because nebulization was supplemented by transtracheal injection of $3 \mathrm{ml}$ lignocaine, which further improved the quality of anesthesia.

Kundraet al. also compared two methods of anesthetizing the airway for awakefiberopticnasotracheal intubation. One of the groups received $4 \mathrm{ml}$ of $4 \%$ lignocaine through nebulization and the other received airway blocks (translaryngeal, bilateral superior laryngeal and lignocaine soaked cotton swabs in the nose). ${ }^{26}$ Although the time taken to intubate was similar in both groups, patients who received lignocaine nebulization for airway anesthesia had to undergo significantly higher stress during the insertion of endotracheal tube through the glottis.

Patient comfort was better in the nerve blocks group as compared with the nebulization group in our study, as deduced by the coughing/gagging episodes as well as the patient assessment of procedure recall. Gupta et al. conducted a study in 2014 which also showed that patient comfort was better in the nerve blocks group as compared with the nebulization group and also vocal cord visibility and ease of intubation as assessed by the bronchoscopist were better in the nerve block group as compared with the nebulization group. ${ }^{10}$ The findings 
Topical Airway Anaesthesia Techniques In Dental Patients With TemporomandibularJoint ..

reported by Reasoneret al. were also similar. ${ }^{25}$ Although, there was no difference in the number of coughing/gagging episodes between the two study groups, patient recall of the procedure was more in the nebulization group. Kundraet al. also reported higher grimace scores, mean HR and BP during insertion of endotracheal tube in patients who received lignocaine via nebulization as compared to nerve blocks. ${ }^{26}$ However, the patient comfort and recall of the procedure were comparable between the two groups.

In our study, vocal cord visibility and ease of intubation as assessed by the bronchoscopist were better in the nerve block group as compared with the nebulization group. This finding is similar to that observed by Graham et al. ${ }^{27}$ They reported that the bronchoscopist preferred transtracheal instillation of LA as compared to LA nebulization or LA instillation through the working port of FOB. However, Reasoner et al. did not find any difference in the quality of airway anesthesia between nebulized LA and nerve blocks as assessed by a blind observer/bronchoscopist. $^{25}$

Finally it would not be wrong to say that both techniques carry their advantages and disadvantages as a stand aloneprocedure. The major advantage of this technique lies in its simplicity and lack of discomfort. In addition, very little working knowledge of the anatomy of theregion is required for its successful implementation. Although this technique may seem ideal, it does have some drawbacks thatlimit its usefulness.

The main disadvantage is that the density of the anaesthesia achievedthroughout the airway is highly variable.Many patients still experience an intact cough reflex, which can make intubation technically challenging. The rate of onset of this technique ishighly dependent on patient compliance.Many patients who need an awake intubation are incapable or unwilling totake deep breaths.

Also, inhalation of local anaesthetic vapours can lead to central nervoussystem depression in patients whose mental status may already bedepressed owing to other disease processes.It should be emphasized that topicalization alone may be inadequate forawake tracheal intubation in some patients as the stretch receptors at theroot of the tongue which cause the gag reflex are submucosal and are noteasily blocked and have to be supplemented by nerve bocks.

Finally the authors would like to stress the limitations of the study. First our research is not completely unbiasedinspite of best efforts. Our second limitation is like any other regional technique; practice will improve the success rate as well as the ability of the practitioner to provide the blocks. Also the plasma levels of lignocaine could not be measured as we do not have this provision at our institute. Because of this limitation we had to depent on the previous limits set on the doses of lignocaine by other authers and constantly fear its toxicity. Further research is needed on this by using higher doses andand newer methods of topicalization. We would also like to emphasize that using a spinal needle for glossopharyngeal nerve block would offer a more clear view of intraoral approach method as suggested by Benumofet al. ${ }^{6}$

In the end we would say that if there is a good possibilitry that intubation and/or ventilation by mask will be difficult,then the airway should be secured while the patient is still awakewith intact reflexes for which nerve blocks would be the best.

\section{Conclusion}

In order for an awake intubation to be successful it is absolutely essential that the patient be properly prepared; otherwise the anaesthesiologist will simplyfulfill a self defeating prophecy. Given the results of the study and the above discussion, the following conclusions may be drawn. The performance of bilateral superior laryngeal, bilateral glossopharyngeal and transtracheal recurrent laryngeal nerve blocks provides adequate airway anesthesia to aid in awake FOB guided intubation. Furthermore, $10 \mathrm{ml}$ of $4 \%$ lignocaine through micronebulizer may not provide acceptable conditions for bronchoscopy, a lower dose of lignocaine through nebulization along with supplemental lignocaine instillation through the working channel of FOB might provide adequate airway anesthesia.

\section{Bibliography}

[1]. El-Sheikh MM, Medra AM. Management of unilateral TM Ankylosis associated with fascial asymmetry. J. Oromaxillofascial surgery 1997 june; 25: (3):109-15.

[2]. AdekeyeEO.Ankylosis of mandible:analyses of 76 cases. J of Oral maxillofacial surgery 1983. July: 41(7):442-9.

[3]. Guven O. Treatment of temporomandibular joint ankylosis by modified fossa prosthesis. J CraniomaxillofacSurg2004; 32:236-42.

[4]. Mishra Manas, BhavsarMrugank, Patel Chinar et al. A comparative study of intubating condition and haemodynamic changes during blind nasal intubation verses fibre optic intubation in cases of temporomandibular joint ankylosis. IJBAR 2014;may:01.

[5]. Erb T, Hampl KF, Schürch M, Kern CG, Marsch SC. Teaching the use of fiberoptic intubation in anesthetized, spontaneously breathing patients. AnesthAnalg. 1999;89:1292-5.

[6]. Benumof JL. Management of the difficult adult airway. With special emphasis on awake tracheal intubation. Anesthesiology. 1991;75:1087-110.

[7]. Barash PG, Cullen BF, Stoelting RK. 5th ed. Philadelphia: Lippincott Williams \& Wilkins; 2006. Clinical Anesthesia; pp. 621-30.

[8]. Ramkumar V. Preparation of the patient and the airway for awake intubation. Indian J Anaesth. 2011;55:442-7.

[9]. Trivedi V, Patil B. Evaluation of airway blocks versus general anesthesia for diagnostic direct laryngoscopy and biopsy for carcinoma of the larynx. A study of 100 patients. Internet J Anesthesiol. 2009;26:1. 
Topical Airway Anaesthesia Techniques In Dental Patients With TemporomandibularJoint ..

[10]. Gupta B, Kohli S, Farooque K, Jalwal G, Gupta D, Sinha S, et al. Topical airway anesthesia for awake fiberoptic intubation: Comparison between airway nerve blocks and nebulized lignocaine by ultrasonic nebulizer. Saudi J Anaesth. 2014;8(Suppl 1):S159.

[11]. Gerheuser F, and Gurtler K, Awake fiberoptic intubation. Anaesthetist 2011 Dec; 60 (12): 1157-74.

[12]. Yu Sun, Jin-Xing Liu, Hong Jiang M, Ye-Sen Zhu M, HuiXu and Yan Huang. Cardiovascular responses and airway complications following awake intubation with blind intubation device and fibre optic bronchoscope: a randomnised controlled study. Eur J. of Anesthesiology2010:27:5: 461-67.

[13]. Murphy P. A fibre-optic endoscope used for nasal intubation. Anesthesia. 1967;22:489-91.

[14]. Cullen L, Taylor D, Taylor S, Chu K. Nebulized lidocaine decreases the discomfort of nasogastric tube insertion: Arandomized, double-blind trial. Ann Emerg Med. 2004;44:131-7.

[15]. Williams KA, Barker GL, Harwood RJ, Woodall NM. Combined nebulization and spray-as-you-go topical local anaesthesia of the airway. Br J Anaesth 2005; 95: 549-53.

[16]. Ovassapian A, Wheeler M. Fiberoptic endoscopy aided technique. In: Benumof JL. Airway management: principle and practice. Missouri: MosbyYearbook; 1996: 282-319.

[17]. Ovassapian A, Yelich SJ, Dykes MH, Brunner EE.Fiberopticnasotracheal intubation - incidence andcauses of failure. AnesthAnalg 1983; 62: 692-5.

[18]. British Thoracic Society Bronchoscopy Guidelines Committee, a Subcommittee of Standards of Care Committee of British Thoracic Society. British Thoracic Society guidelines on diagnostic flexible bronchoscopy. Thorax 2001; 56(Suppl 1): 1-21.

[19]. Bromage PR, Robson JG. Concentrations of lignocaine in the blood after intravenous, intramuscular epidural and endotracheal administration. Anaesthesia 1961; 16: 461-78.

[20]. Webb J. Local anaesthesia for fibreoptic bronchoscopy- where are we now? Respir Med 1990; 84: $349-50$.

[21]. Langmack EL, Martin RJ, Pak J, Kraft M. Serum lidocaine concentrations in asthmatics undergoing research bronchoscopy. Chest. 2000;117:1055-60.

[22]. Wu FL, Razzaghi A, Souney PF. Seizure after lidocaine for bronchoscopy: Case report and review of the use of lidocaine in airway anesthesia. Pharmacotherapy. 1993;13:72-8.

[23]. Ovassapian A, Yelich SJ, Dykes MH, Brunner EE. Blood pressure and heart rate changes during awakefiberopticnasotracheal intubation. AnesthAnalg. 1983;62:951-4.

[24]. Techanivate A, Leelanukrom R, Prapongsena P, Terachinda D. Effectiveness of mouthpiece nebulization and nasal swab stick packing for topical anesthesia in awake fiberopticnasotracheal intubation. J Med Assoc Thai. 2007;90:2063-71.

[25]. Reasoner DK, Warner DS, Todd MM, Hunt SW, Kirchner J. A comparison of anesthetic techniques for awake intubation in neurosurgical patients. J NeurosurgAnesthesiol. 1995;7:94-9.

[26]. Kundra P, Kutralam S, Ravishankar M. Local anesthesia for awakefibreopticnasotracheal intubation. ActaAnaesthesiol Scand. 2000;44:511-6.

[27]. Graham DR, Hay JG, Clague J, Nisar M, Earis JE. Comparison of three different methods used to achieve local anesthesia for fiberoptic bronchoscopy. Chest. 1992;102:704-7.

[28]. Chatrath V, Sharan R, Jain P, et al. The efficacy of combined regional nerve blocks in awakeorotrachealfiberoptic intubation. Anesthesia, Essays and Researches. 2016;10(2):255-261. 